\title{
Space Mission Characteristics and Requirements to be Addressed by Space-Data Router Enhancement of Space-Data Exploitation
}

\author{
Ioannis A. Daglis ${ }^{1}$, Olga Sykioti ${ }^{1}$, Anastasios Anastasiadis ${ }^{1}$, Georgios Balasis ${ }^{1}$, \\ Iphigenia Keramitsoglou ${ }^{1}$, Dimitris Paronis ${ }^{1}$, Athanassios Rontogiannis ${ }^{1}$, \\ and Sotiris Diamantopoulos ${ }^{2}$ \\ ${ }^{1}$ National Observatory of Athens, Athens, Greece \\ \{daglis, sykioti, anastasi, gbalasis,ikeram, paronis, tronto\}@noa.gr \\ ${ }^{2}$ Democritus University of Thrace, Greece \\ sdiaman@ee.duth.gr
}

\begin{abstract}
Data distribution and access are major issues in space sciences as they influence the degree of data exploitation. The project "Space-Data Routers" (SDR) has the aim of allowing space agencies, academic institutes and research centres to share space data generated by single or multiple missions, in an efficient, secure and automated manner. The approach of SDR relies on space internetworking - and in particular on Delay-Tolerant Networking (DTN), which marks the new era in space communications, unifies space and earth communication infrastructures and delivers a set of tools and protocols for space-data exploitation. The project includes the definition of limitations imposed by typical space mission scenarios in which the National Observatory of Athens (NOA) is currently involved, including space exploration, planetary exploration and Earth observation missions. In this paper, we present the mission scenarios and the associated major SDR expected impact from the proposed space-data router enhancements.
\end{abstract}

Keywords: space science, spacecraft data distribution, internetworking, space communications.

\section{Introduction}

Vast quantities of space data have to be transferred from space to the operation centres and, beyond, to the research institutions in order to be analysed and exploited. The basic aim of Space Data Routers project is to allow space agencies, universities and research centres to share space data generated by a single or multiple missions, in a more flexible, secure and automated manner. Currently, efficient space-data exploitation faces two major obstacles: Firstly, research institutions have limited access to scientific data since their limited connectivity time to satellites directly confines their scientific capacity. Secondly, space-data collection centres, such as ESOC, lack sufficient mechanisms for efficient communication with interested end-users, let alone the lack of mechanisms for efficient data dissemination. The result is frequently quite 
disappointing: space data remain stored and unexploited, until they become obsolete or useless and consequently are being removed. In the context of space-data exploitation, the situation is expected to aggravate in the near future: space data volume will increase (consider the upcoming Sentinel missions, for example), but the mechanisms for disseminating and exploiting data are not yet in place. Therefore, the efficient exploitation and dissemination of space data should not be considered as a peripheral issue, but rather as an important missing mechanism from the European Infrastructure. The Space-Data Router implements a dual role: It increases communication flexibility in Space and forms a mission-/application-oriented communication overlay for data dissemination, on Earth. These goals will be realized in three stages:

- Design and implementation of a Space-Data Router; a crucial component for space internetworking.

- Integration of the Space-Data Router within a core existing testbed, tested and evaluated in terms of specific space mission scenarios' requirements, overlay architectural design, compatibility with ESA equipment, protocols and policies, scalability in communicating with deep-space components, and interoperability with NASA's equipment.

- Development of a pilot application to integrate thematically various practical space mission scenarios. A cross-mission approach will benefit scientific centres worldwide and also allow for more accurate and timely data analysis. The application will allow for investigating in depth the potential of creating thematically-oriented space-data overlays in the future.

The application scenarios, presented in this paper, have been properly selected to match with the scientific objectives of the project. The major scientific objectives along with the corresponding scenarios are for Space Data Routers to:

- Boost the dissemination capability for space data on Earth by extending end user access to space data through communicating Ground Stations and Space Research Centres.

- Allow for exploiting data from Deep Space and disseminating them naturally through unified communication channels.

- Exploit European Scientific Capacity as well as ESA's existing infrastructure, resources, protocols policies and assets.

- Allow for cross-mission scientific applications, by demonstrating the capability of the DTN space-data overlays to administer thematic cross-mission space-data.

The original scenarios include operational and application information, as they constitute the reference of comparison for the new architecture [14]. Additionally, the expected impact on each scenario by using the Space Data Routers based architecture is foreseen from the proposed SDR enhancement. In a later stage of the project, this impact will be evaluated in terms of data transfer, dissemination capacity, and operative burden.

\section{Space-Data Routers Enhancements}

The main advantage of the Space-Data Router is that it operates on top of existing network protocols and technologies, creating a DTN overlay [3] that interconnects networks with very diverse characteristics, such as space and terrestrial. Therefore 
DTN provides the basic functionality for efficient space-to-earth data dissemination. In addition the router is developed on top of real space protocols allowing for the direct interoperation with current space infrastructure. Furthermore, a sophisticated application will also be implemented in order to support, highlight and assess system's capabilities.

A novel routing mechanism is being implemented to support communications in Space and on Earth. More specifically, we are designing a routing algorithm that is based on Contact Graph Routing algorithm [2] with several additions and modifications. In particular, we propose a cost calculation function that takes into account parameters such as the bandwidth, the delay, the financial cost, the node storage capability, and the node or owner agency's trustability. These parameters are included into the routing decision mechanism with specific weights that are configured based on specific mission objectives. For example, in a transmission session of vast amounts of data, storage limitations of intermediate nodes is an important parameter that should be given more weight than others. In essence, data will be optimally routed towards their final destination, depending on the specificities of each mission and each scientific instrument.

As far as the actual transmission of data is concerned, sophisticated transport protocols, such as LTP [12], are used to mitigate losses due to error-prone deep-space communication channels. Furthermore, we are in the process of designing and implementing a protocol for Delay Tolerant Payload Conditioning (DTPC), which is used as an end-to-end protocol on top of the DTN Architecture. DTPC protocol complements the DTN services offered by the Bundle Protocol [16] and operates only at the endpoints of the communication system enabling end-to-end services, such as application data aggregation and elision, in-order delivery of application data, end-to-end retransmission-based reliability and duplicate suppression.

Finally, a major technical contribution of the Space-Data Routers project is the implementation of a web-based application that interconnects data providers, user institutions, infrastructure providers and administrators. A graphical user interface will be developed, to facilitate easy and automated access to the application database. Given the wide user community, specific focus is given on providing a user-friendly environment that will allow for fast browsing between mission profiles. Furthermore, the cross-mission categorization will facilitate the interdisciplinary approach on many data sets.

\section{Application Scenarios}

\subsection{Demonstrate the Capability of the Space-Data Routers to Extend End-User Access to Space Data. Case: Combining Real-Time/Archived AVHRR HRPT Data from Local HRPT Receiving Stations Worldwide}

\section{Description.}

The Advanced Very High Resolution Radiometer (AVHRR), aboard the NOAA meteorological satellites, is a cross-track scanning system with five spectral bands having a ground resolution of $1.1 \mathrm{~km}$ and a frequency of earth scans twice per day. Each 
pass of the satellite provides a $2399 \mathrm{~km}$ wide swath. AVHRR data are used for retrieving various geophysical parameters such as sea surface temperatures, energy budget, and vegetation content. Through the High Rate Picture Transmission (HRPT) service $(1700 \mathrm{MHz}$, at a transmission rate of $665,400 \mathrm{bps}$ ) installed on the NOAA satellites, user stations throughout the world can acquire data from three or more consecutive overpasses. In the specific scenario, as a demonstration, AVHRR data will be gathered from various ground stations and disseminated as a composite dataset in real-time via network nodes, which will incorporate the concepts and protocols of Delay Tolerant Networking. This scheme is similar to the one described in the currently running WMO's (World Meteorological Organization) RARS project which is focused on delivering NOAA ATOVS data (AVHRR and ATOVS sensors are both mounted aboard NOAA polar satellites) within no more than 30 minutes from acquisition [13].

\section{SDR Expected Impact.}

The expected impact is the increase of data availability and delivery throughput for real-time access of satellite data. Moreover, the deployment of the DTN nodes is expected to contribute to an effective utilization of the ground communication infrastructures, enhancing thus the data sharing mechanisms, circumventing the downlink constraints. At the same time, the scalability potential of the SDR concept will be assessed. Applicability of the approach to other types of direct readout broadcasting systems (e.g. MODIS) will be further examined.

\subsection{Demonstrate the Potential of Exploiting Data from Deep Space and Disseminate It Naturally through Unified Communication Channels. Case: Hyperspectral Images Captured by MEx/OMEGA}

\section{Description.}

MEx (Mars Express) is the first 'flexible' mission of ESA's long-term science exploration programme. Hyperspectral images are captured by the OMEGA sensor on-board MEx. The OMEGA data type is a hyperspectral image cube of $~ 120$ Mbytes size [5]. The European Space Operations Control Centre (ESOC) in Darmstadt communicates with MEx via ESA's New Norcia ground station (DSA-1) in Perth, Australia [7]. DSA-1 coverage depends on the actual distance between Mars and Earth. The Mars visibility window at DSA-1 is of the order of 10-12 hours. Within this visibility period there is a nominal tracking time of 8 hours per day, in which MEx communicates with DSA-1. Downlink communication sessions are continuous. Data are then transferred from ESOC/DSA-1 to IAS (Institut d'Astrophysique Spatiale, France) on a dedicated server, where they are decompressed, Planetary Data System (PDS) formatted and archived. Data processing is restricted to the IAS team during a proprietary six months period. After this period, the calibrated corrected image cubes become available to the scientific community through the ESA Planetary Science Archive. Currently, 5-6 image cubes are received per year at NOA via a slow ftp connection. 


\section{SDR Expected Impact.}

The use of the proposed SDR architecture for MEx/OMEGA data is expected to:

- Increase the data volume received from Mars Express, by increasing the connectivity and downlink time of Mars Express with ground stations.

- Provide access to current (possibly raw) image cubes.

- Increase the access speed to high-volume data.

\subsection{Demonstrate the Sufficiency of DTN Space-Data overlays to Administer Thematic Cross-Mission Space Data}

\section{Case 1: Multi-mission Study of the Sun-Earth Connection}

\section{Description.}

The term "space weather" refers to conditions on the Sun and in the solar wind, Earth's magnetosphere, ionosphere, and thermosphere that can influence the performance, efficiency, and reliability of space- and ground-based infrastructure and can endanger unprotected humans in space conditions or above the Earth's poles. Nowadays, information from a single spacecraft vantage point can be replaced by multispacecraft distributed observatory methods and adaptive mission architectures that require computationally intensive analysis methods. Future explorers far from Earth will be in need of real-time data assimilation technologies to predict space weather at different solar system locations.

\section{SDR Expected Impact.}

The main requirements for this application scenario are the real-time availability of electric field, magnetic field and charged particle data as recorded by multiple missions in geospace and in the solar wind. The use of the DTN architecture could provide/improve:

- Real-time data acquisition from multiple missions for monitoring ULF/VLF wave occurrence and its effects on radiation belt dynamics.

- Successful data transmission even in harsh/challenged communication conditions.

The objective of this scenario is to now-cast and, ultimately, forecast the influence of solar disturbances (which propagate through interplanetary space and impinge on the terrestrial magnetosphere) on the development of electromagnetic waves in the magnetosphere and the wave effect on radiation belt variability.

We plan to test the capability of Space-Data Routers to efficiently distribute to registered end-users the relevant data streams from an ongoing NASA mission (THEMIS), an ESA mission (Cluster) and an upcoming NASA mission (Radiation Belt Storm Probes) [1,6]. The simultaneous real-time sampling of space plasmas from multiple points with cost-effective means and measuring of phenomena with higher resolution and better coverage will further address outstanding science questions. 


\section{Case 2: Land Surface Temperature}

\section{Description.}

"LST" is a multi mission, single parameter case study. Knowledge of surface temperature and its temporal and spatial variations within a city environment is of prime importance to the study of urban climate and human-environment interactions $[10$, $11,17,18]$. For the purposes of the SDR project, the satellites that carry thermal infrared sensors useful for the study of LST distribution are considered. Overall, three different spatial resolutions of $3 \mathrm{~km}, 1 \mathrm{~km}$ and $100 \mathrm{~m}$, respectively, provide a different perspective to the study and characterization of the Urban Heat Island (UHI) phenomenon. In particular, $1 \mathrm{~km}$ spatial and few images per day temporal resolution (e.g. MODIS, AVHRR and (A)ATSR) is an adequate compromise which gives the general picture of the hot spots and relevant patterns at a regional scale. If one wishes to investigate the phenomena in a finer scale, then one should use the high-resolution images (90/120m, e.g. Landsat TM and ASTER) for local/municipality level studies for long-term planning. However, the diurnal variation of the phenomenon is only possible with geostationary satellites (MSG-SEVIRI). Currently, one of the main problems is the different location of the data as they come from different providers.

\section{SDR Expected Impact.}

- The application allows for data gathering from multiple missions for one scientific objective

- Same storage location for all data as well as for real time and on demand.

- Ensure real-time data acquisition.

\subsection{Demonstrate the Capability of Space-Data Routers to Deliver Efficiently to End-Users Vast Volumes of Data over Terrestrial Internetworks. Case: New Deployments in Space: Sentinels}

\section{Description.}

This is an upcoming mission scenario. At the moment (2012), ESA is developing five new missions called Sentinels. Each Sentinel mission is based on a constellation of two satellites to fulfil the revisit and coverage requirements to provide robust datasets for the Global Monitoring for Environment and Security (GMES) Services. This scenario focuses on two Sentinels, Sentinel-1 corresponding to C-band synthetic aperture radar (SAR) applications [8,9] and Sentinel-2, which will provide high-resolution optical observation for land and emergency services [15]. The GMES Sentinels Flight Operations Segment (FOS) is being established at ESOC, Darmstadt, Germany. The Payload Data Ground Segment (PDGS) operation baseline imposes strong constraints on the product timeliness provided to end-users. The PDGS is designed as a network of distributed ground stations and complementary centres imposing product data exchanges amongst PDGS remote locations and between the PDGS and its users. This implies an adequate dimensioning of internal data circulation resources between centres (electronically or else), complemented by data dissemination resources between the various archive locations and end users (electronic only). 


\section{SDR Expected Impact.}

The challenge of this scenario is that it refers to an upcoming mission and it calls for the systematic acquisition with a couple of twin satellites of all land surfaces. On ground, this implies a very large data volume to manage with appropriate processing, archiving and networking resources. As this data will be used, among other operation modes, for emergency situations SDR will be called to distribute vast volumes of data over terrestrial internetworks to the appropriate user locations on time.

\section{Summary}

The ultimate goal of "Space-Data Routers" is to boost collaboration and competitiveness of European Space Agency, European Space Industry and European Academic Institutions towards an efficient architecture for exploiting space data. The proposed approach in this project relies on space internetworking - and in particular in DelayTolerant Networking (DTN), which marks the new era in space communications, unifies space and earth communication infrastructures and delivers a set of tools and protocols for space-data exploitation within a single device.

Acknowledgements. The project "Space-Data Routers for Exploiting space data" has received funding from the European Community's Seventh Framework Programme (FP7-SPACE-2010-1, SP1 Cooperation, Collaborative project) under grant agreement $\mathrm{n}^{\circ} 263330$. This paper reflects only the authors' views and the Union is not liable for any use that may be made of the information contained therein.

\section{References}

1. Angelopoulos, V.: The THEMIS Mission. Space Sci. Rev. 141, 5-34 (2008)

2. Burleigh, S.: Dynamic Routing for Delay-Tolerant Networking in Space Flight Operations. In: SpaceOps 2008 Conference on Protecting the Earth, Exploring the Universe, Heidelberg (2008)

3. Cerf, V., Burleigh, S., Torgerson, L., Durst, R., Scott, K., Fall, K., Weiss, H.: DelayTolerant Network Architectur. IETF RFC 4838, Internet Engineering Task Force (April 2007), http: / /www. ietf.org/rfc/rfc4838.txt

4. Daglis, I.A. (ed.): Space storms and space weather hazards. Kluwer, Dordrecht (2001)

5. ESA Mars Express Operations, http://sci.esa.int/science-e/www/area/index.cfm?fareaid=9

6. Escoubet, C.P., Fehringer, M., Goldstein, M.: Introduction: The Cluster mission. Annales Geophysicae 19, 1197-1200 (2001)

7. ESA Operations and Situational Awareness - Mars Express operations, http: / / www. esa.int/esaMI / Operations / SEMORMQJNVE_0.html

8. GMES Space Component Sentinel-1 Payload Data Ground Segment System Technical Budget, GMES-GSEG-EOPG-TN-08-0011, ESA (2009)

9. GMES Space Component Sentinel-1, Payload Data Ground Segment (PDGS) and Operations Concept Document, GMES-GSEG-EOPG-TN-08-0012, ESA (2010) 
10. Hung, T., Uchihama, D., Ochi, S., Yasuoka, Y.: Assessment with satellite data of the urban heat island effects in Asian mega cities. International Journal of Applied Earth Observation and Geoinformation 8, 34-48 (2006)

11. Keramitsoglou, I., Kiranoudis, C.T., Ceriola, G., Weng, Q., Rajasekard, U.: Identification and Analysis of Urban Surface Temperature Patterns in Greater Athens, Greece, Using MODIS Imagery. Remote Sensing of Environment 115, 3080-3090 (2011)

12. Ramadas, M., Burleigh, S., Farrell, S.: Licklider Transmission Protocol - Specification. IETF RFC 5326, experimental (2008),

http: / / www.ietf.org/rfc/rfc5326.txt

13. Regional ATOVS Retransmission Services (RARS), World Meteorological Organization, http: / / www.wmo.int/pages/prog/sat/rars_en.php

14. Scenario Requirements Report, Space-Data Routers (March 2011), http: / /www. spacedatarouters.eu/wpcontent/uploads/2010/12/D21.pdf

15. Sentinel-2 Payload Ground Segment, System Technical Budget Document, GMES-GSEGEOPG-TN-09-0031, ESA and Operations Concept Document, GSEG-EOPG-TN-09-0008, ESA (2010)

16. Scott, K., Burleigh, S.: Bundle Protocol Specification. IETF RFC 5050, Internet Engineering Task Force (November 2007), http: / /www . ietf . org/rfc/rfc4838. txt

17. Stathopoulou, M., Cartalis, C.: Downscaling AVHRR land surface temperatures for improved surface urban heat island intensity estimation. Remote Sensing of Environment 113, 2592-2605 (2009)

18. Weng, Q.: Thermal infrared remote sensing for urban climate and environmental studies: Methods, applications, and trends. ISPRS Journal of Photogrammetry and Remote Sensing 64, 335-344 (2009) 